\title{
A 250 GHz PLANAR LOW NOISE SCHOTTKY RECEIVER
}

\section{Walid Y. Ali-Ahmad, ${ }^{1}$ William L. Bishop, ${ }^{2}$ Thomas W. Crowe, ${ }^{2}$ and Gabriel M. Rebeiz}

\author{
${ }^{1}$ NASA/Center for Space Terahertz Technology \\ Electrical Engineering and Computer Science Department \\ University of Michigan \\ Ann Arbor, Michigan 48109-2122 \\ ${ }^{2}$ Semi-Conductor Device Laboratory \\ Electrical Engineering Department \\ University of Vinginia \\ Charlottesville, Virginia 22901
}

Received January 17, 1993

\begin{abstract}
A planar quasi-optical Schottky receiver based on the quasi-integrated horn antenna has been developed and tested over the $230-280 \mathrm{GHz}$ bandwidth. The receiver consists of a planar GaAs Schottky diode placed at the feed of a dipole-probe suspended on a thin dielectric membrane in an etched-pyramidal horn cavity. The diode has a $1.2 \mu \mathrm{m}$ anode diameter and a low parasitic capacitance due to the use of an etched surface channel. The antenna-mixer results in a measured DSB conversion loss and noise temperature at $258 \mathrm{GHz}$ of $7.2 \mathrm{~dB} \pm 0.5 \mathrm{~dB}$ and $1310 \mathrm{~K} \pm 70 \mathrm{~K}$, respectively, at room temperature. The design is compatible with SIS mixers, and the low cost of fabrication and simplicity makes it ideal for submillimeter-wave imaging arrays requiring a $10-20 \%$ bandwidth.
\end{abstract}




\section{INTRODUCTION}

Fundamental waveguide mixers using whisker-contacted Schottky diodes are currently used in room temperature submillimeter-wave receivers. Although whisker-contacted diodes do not suffer from a large parasitic capacitance and waveguide mixers can be mechanically tuned for the optimium performance, the waveguide mixer and associated corrugated-horn antenna are very expensive to machine for frequencies above $200 \mathrm{GHz}$. Integrated receivers consisting of a planar antenna and a planar diode are easier to manufacture, smaller, and much less expensive than waveguide mixers when produced in large quantities. They can also be arrayed for imaging applications without a substantial increase in cost.

A planar low noise receiver for the millimeter-wave region was developed and tested by Ali-Ahmad et al. [1]. The receiver is tunerless and its performance compared favorably with the best wideband tunerless waveguide mixers over the $86-106 \mathrm{GHz}$ frequency range. The quasi-integrated horn antenna used in the receiver has a directivity of $20 \mathrm{~dB}$, a high Gaussian coupling efficiency and a wide bandwidth $(20 \%)$. The dipole-probe suspended on the membrane inside the horn is designed so that its impedance offers a close conjugate match to the diode impedance, eliminating the need for an RF matching network. This results in a wideband mixer circuit that forms an integral part of the antenna structure. In this work, we present a $250 \mathrm{GHz}$ receiver based on the quasi-integrated horn antenna [1]. The receiver can also be fabricated using SIS junctions and offers a practical solution for submillimeter-wave imaging arrays.

\section{ANTENNA AND MIXER DESIGN}

A quasi-integrated horn antenna has been designed by Eleftheriades et al. [2] to overcome the limitations of the large flare angle of the integrated horn antenna introduced in $[3,4]$. In this design, a flared machined section is attached to the front part of the integrated horn antenna to result in higher gain patterns (Fig. 1). The abrupt change of flare-angle at the junction of the integrated section and the machined section of the horn acts as a mode converter that excites mainly the $\mathrm{TE}_{10}, \mathrm{TE}_{12} / \mathrm{TM}_{12}$ and $\mathrm{TE}_{30}$ modes. 
These modes are properly phased on the radiating aperture by appropriately selecting the length and the flare-angle of the machined section. Table I shows the theoretical gain and Gaussian coupling efficiency from $222 \mathrm{GHz}$ to $279 \mathrm{GHz}$ of a $20 \mathrm{~dB}$ quasi-integrated horn antenna. It is seen that the antenna is wideband in its Gaussian coupling efficiency. The measured patterns at the design frequency of $250 \mathrm{GHz}$ and at $237 \mathrm{GHz}$ and $279 \mathrm{GHz}$ are shown in figures $2 \mathrm{a}$ and $2 \mathrm{~b}$, respectively.

\section{Table I}

\begin{tabular}{||c||c|c|c|c|c||}
\hline Frequency (GHz) & 222 & 237 & 250 & 258 & 279 \\
\hline Gaussian Coupling Efficiency (\%) & 86 & 95 & 97 & 97 & 92 \\
\hline Directivity (dB) & 18.7 & 19.4 & 20.0 & 20.4 & 21.3 \\
\hline
\end{tabular}

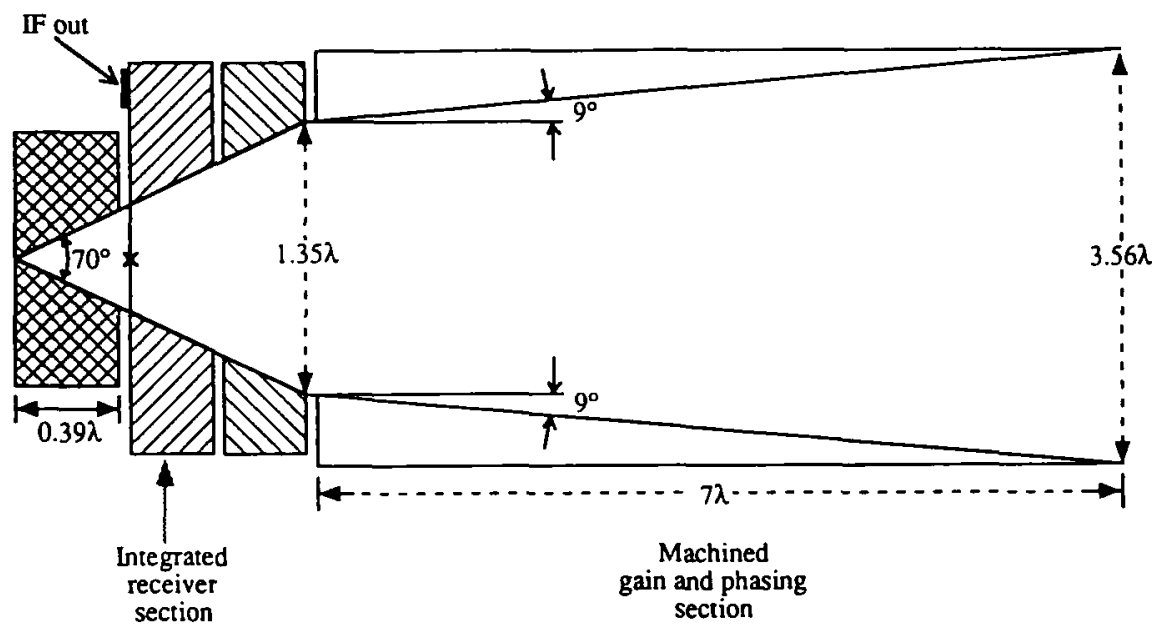

Figure 1: The 20dB quasi-integrated horn antenna. 


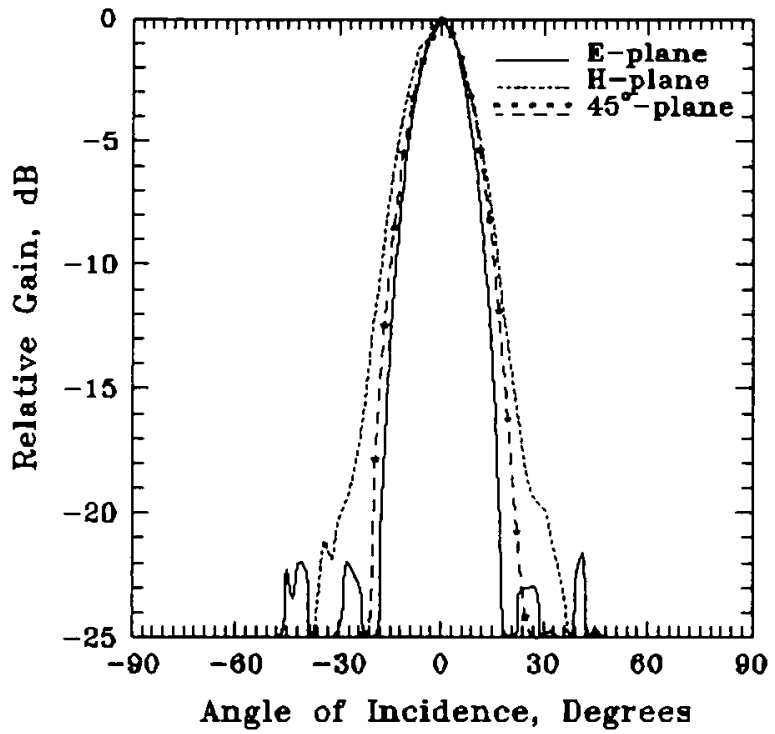

(a)

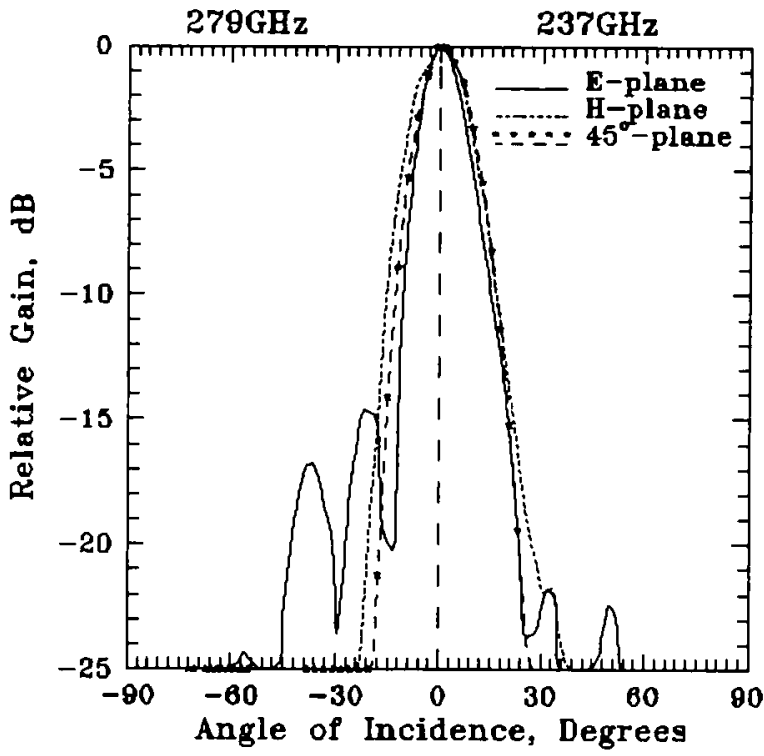

(b)

Figure 2: The measured $\mathrm{E}-, \mathrm{H}-$, and $45^{\circ}$-plane patterns of the quasiintegrated antenna at $250 \mathrm{GHz}(\mathrm{a})$, and $237 \mathrm{GHz}$ and $279 \mathrm{GHz}$ (b). 
The quasi-integrated receiver consists of an integrated section (Fig. 3) and a machined section which is attached to the front of the integrated horn antenna (not shown in Fig. 3). The integrated structure is made of low and high resistivity silicon wafers etched anisotropically and stacked together to form a pyramidal cavity with a $70^{\circ}$ flare angle [3]. The low resistivity wafers are used as mechanical wafers. The mixer circuitry is integrated on the high resistivity wafers. The feed-dipole with the CPS lines is integrated on the membrane wafers as shown in Fig. 4. The horn sidewalls of each silicon wafer, except walls A and B (Fig. 3), are coated with $0.5 \mu \mathrm{m}$ thick gold by thin-film evaporation. Wall $A$ is left uncoated so as not to short the IF and RF signals on the CPS line. A V-shaped cavity is etched in wall B in order to eliminate any radiation from the CPS line. The height of the $\mathrm{V}$-shaped groove is $140 \mu \mathrm{m}$ which is seven times larger than the separation between the two strips forming the CPS line and therefore wall B does not affect the impedance of the CPS line. The cavity is small enough so that no higher-order modes are present at $250 \mathrm{GHz}$. The absence of gold on wall $\mathrm{A}$ and the groove in wall $\mathrm{B}$ result in a $1.2-1.3 \mathrm{~dB}$ measured $\mathrm{RF}$ loss using a microwave model of the integrated structure at $2.5 \mathrm{GHz}$. The uncoated wall $A$ and the groove in wall $B$ have no effect on the far-field patterns (as shown in Fig. 2).

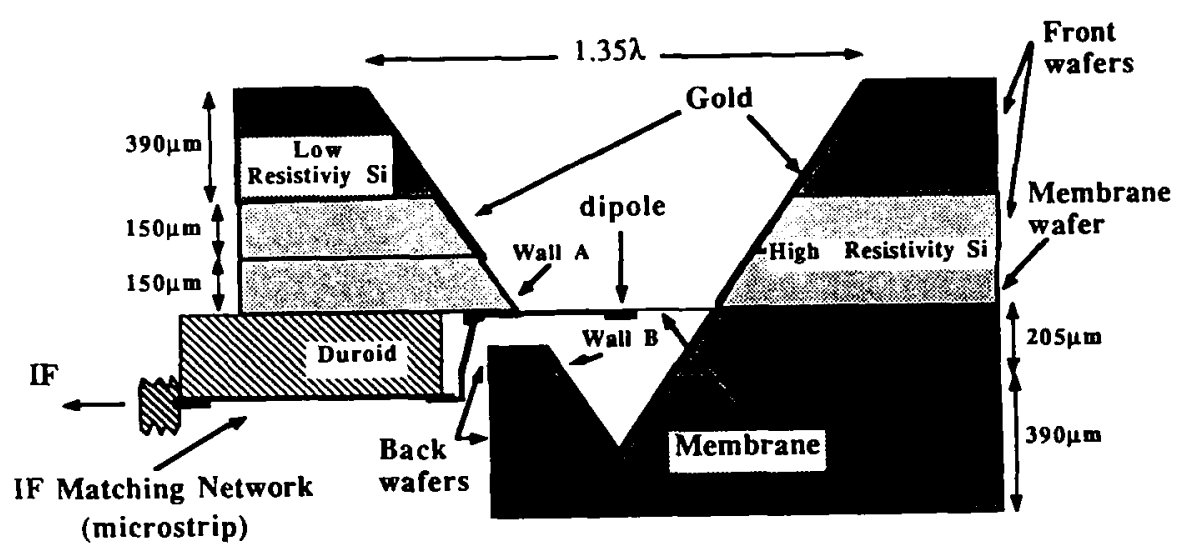

Figure 3: The integrated horn antenna receiver structure. 
In the mixer design (Fig. 4), the length of the feed-dipole and its position inside the integrated horn antenna are chosen to result in a diode impedance that is a close conjugate match to the RF diode impedance [4]. The planar diode is therefore epoxied right at the dipole apex without any additional RF matching network. The separation between the two strips of the CPS line on the silicon wafer is $20 \mu \mathrm{m}$ rather than $40 \mu \mathrm{m}$ on the membrane in order to minimize any radiation losses of the CPS line. The CPS line impedance is $240 \Omega$ on the membrane and $85 \Omega$ on the silicon substrate. The capacitor integrated on the membrane is $53 \mathrm{fF}$ approximately and the one integrated on silicon is $64 \mathrm{fF}$. This results in a LPF with a $-3 \mathrm{~dB}$ corner frequency of $70 \mathrm{GHz}$ and a rejection of $-26 \mathrm{~dB}$ at $250 \mathrm{GHz}$. A microstrip quarter-wave transformer is fabricated on a Duroid 5870 substrate [5] and used to match the $1.4 \mathrm{GHz}$ IF diode output impedance to $50 \Omega$. The IF matching network is specifically not integrated on the high resistivity silicon substrate to facilitate the use of different IF matching networks.

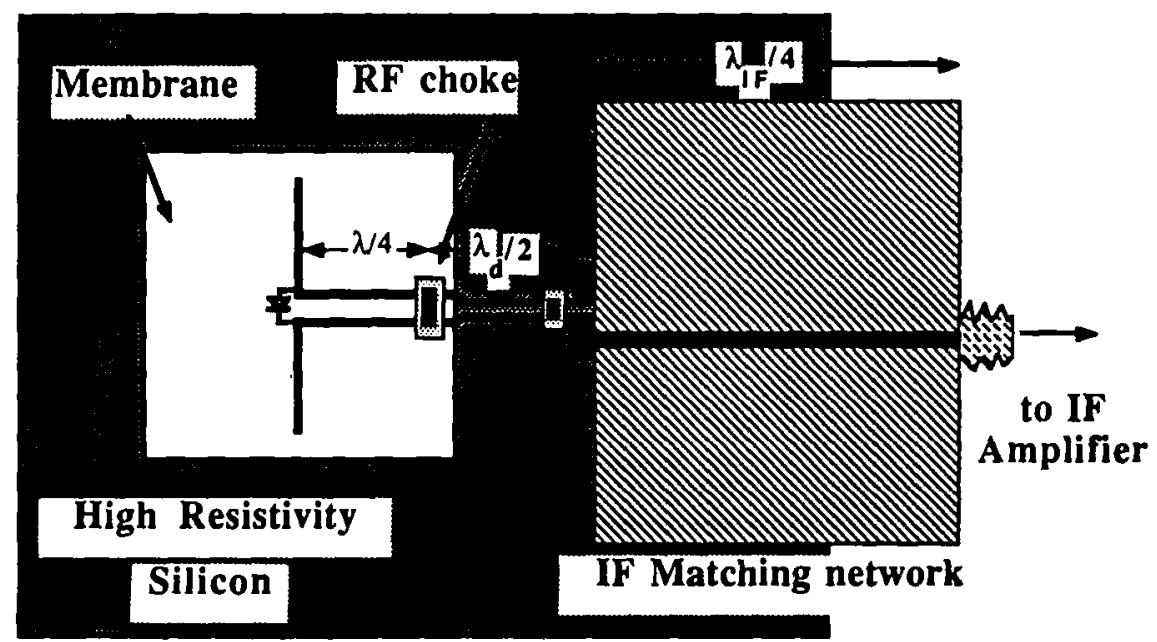

Figure 4: The mixer design. 
The mixer diode is the UVA SC1T4-S20 planar Schottky diode with $1.2 \mu \mathrm{m}$ anode diameter, a $2-3 \mathrm{fF}$ zero-bias junction capacitance, a $5-6 \mathrm{fF}$ parasitic capacitance, an ideality factor of 1.12 and a $9-11 \Omega \mathrm{DC}$ series resistance. This diode has been used before in a submillimeter-wave wavegude mixer and resulted in a $1370 \mathrm{~K}$ DSB noise temperature at $345 \mathrm{GHz}$ [6]. The $5-6 \mathrm{fF}$ parasitic capacitance is reduced to $2-3 \mathrm{fF}$ by chemically thinning the diode before mounting it from a thickness of $50 \mu \mathrm{m}$ to a thickness of $20 \mu \mathrm{m}$. The reflection algorithm developed by Kerr et al. [7] predicts a diode impedance of $65-\mathrm{j} 30 \Omega$ at $250 \mathrm{GHz}$. By using a $1.35 \mathrm{GHz}$ microwave model of the receiver structure shown in Fig. 3 and modeling the thinned diode using a stycast block on the dipole feeds, we obtain a close conjugate match to the diode RF impedance with a $0.39 \lambda$ - long dipole positioned $0.38 \lambda$ from the apex. The measured input impedance on the scale model is $60+j 25 \Omega$ at the design frequency including the effect of the uncoated sidewall (wall A), the V-shaped groove (wall B), and the stycast block. Figure 5 shows the feed-dipole impedance over a $20 \%$ bandwidth.

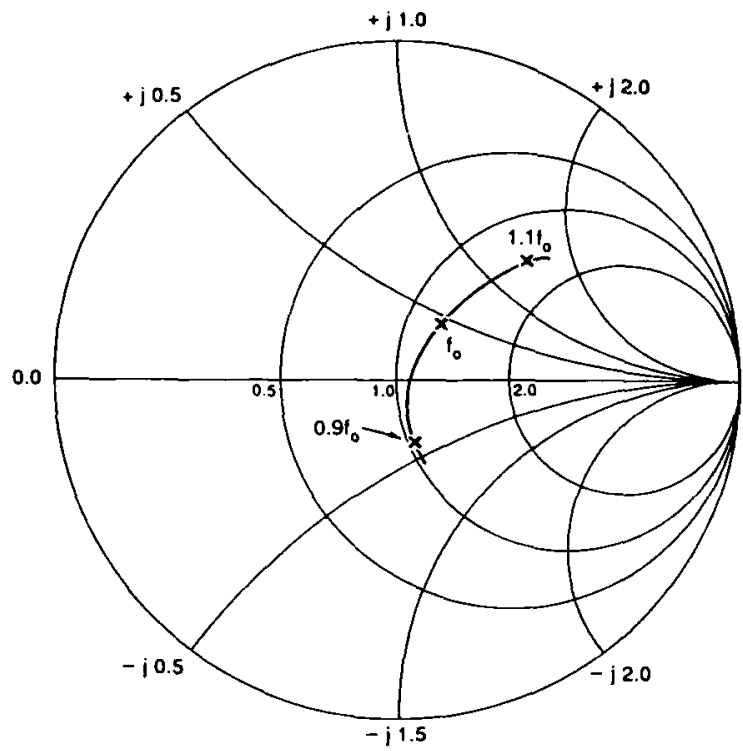

Figure 5: The measured feed-dipole impedance at $250 \mathrm{GHz}$ over a $20 \%$ bandwidth using a $1.35 \mathrm{GHz}$ model of the integrated horn antenna structure. 


\section{RECEIVER MEASUREMENTS}

\section{Video Detection:}

Video detection measurements were done from $230 \mathrm{GHz}$ to $280 \mathrm{GHz}$ (see [1] for more detail). The video responsivity is defined as the ratio of the detected low-frequency voltage across a $120 \mathrm{k} \Omega$ load over the total RF plane-wave power incident on the quasi-integrated horn antenna aperture. A $3 \mathrm{~mm} \times 3 \mathrm{~mm}$ bismuth bolometer is used to measure the incident power density on the antenna aperture [8]. The area of the bolometer is larger than $2 \lambda \times 2 \lambda$ at the measurement frequencies. The video responsivity varied around $450 \mathrm{~V} / \mathrm{W}$ or $82 \mathrm{mV} /\left(\mathrm{mW} / \mathrm{cm}^{2}\right)$ for a $3-6 \mu \mathrm{A} \mathrm{dc}$ bias current (Fig. 6). The variation in the data is due to the $\pm 5 \%$ accuracy in the power measurements and the variation in the feed-dipole impedance. Excluding the $2.0 \mathrm{~dB}$ loss resulting from the $62.5 \%$ aperture efficiency (coupling efficiency of the quasiintegrated horn antenna to a plane wave) and the 1.2dB RF-loss due to the uncoated wall $A$, the measurements result in a diode video responsivity of $1000 \mathrm{~V} / \mathrm{W}$ referred to the feed-dipole terminals. This is competitive with the performance of whisker-contacted diodes at 250 $\mathrm{GHz}$ in a WR-03 waveguide.

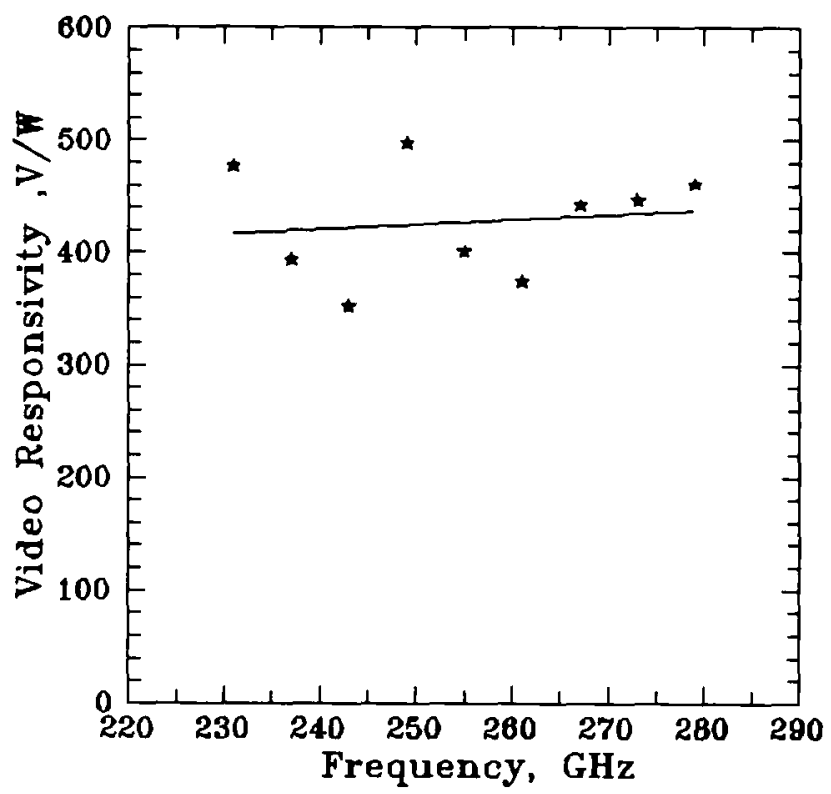

Figure 6: The measured video responsivity of the antenna-mixer from $230 \mathrm{GHz}$ to $280 \mathrm{GHz}$. 


\section{Heterodyne Detection:}

The double-sideband conversion loss and noise temperature of the mixer are measured using a hot/cold load method. A Mach-Zender interferometer is used to combine the RF signal and the LO signal. Receiver measurements were done at room temperature over the $230-280 \mathrm{GHz}$ frequency range. At each frequency, the optimum LO power is approximately $2.5 \mathrm{~mW}$ available at the quasi-integrated antenna aperture, and the optimum dc biasing is around $1.4 \mathrm{~mA}$. The LO power at the antenna aperture was estimated by measuring the transmitted power from the tripler using the large-area bolometer technique described in [8], and reducing it by the RF loss in the quasi-optical path between the LO port and the integrated receiver. During measurements, the loss due to the non-zero IF reflection coefficient at the mixer output port was between $0.5 \mathrm{~dB}$ and $0.55 \mathrm{~dB}$.

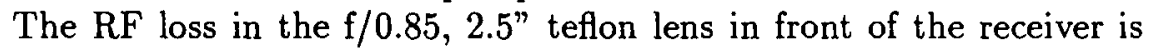
estimated to be $0.3 \mathrm{~dB}$ due to reflection loss and $0.3-0.4 \mathrm{~dB}$ due to dielectric loss (obtained from $[9,10]$ ). The RF diplexer loss is estimated to be 0.1-0.2dB. The IF mismatch and RF path losses are removed from the receiver measurements [1], and the double sideband conversion loss and noise temperature are shown in Fig. 7. The conversion loss includes the antenna Gaussian coupling loss, any power loss in the receiver structure, the RF mismatch between the feed-dipos and diode impedances, and the diode intrinsic conversion loss. A minimum antenna-mixer DSB conversion loss of $7.2 \mathrm{~dB} \pm 0.5 \mathrm{~dB}$ and noise temperature of $1310 \mathrm{~K} \pm 70 \mathrm{~K}$ are obtained at $258 \mathrm{GHz}$. It is seen that the receiver has a comfortable $10 \%$ bandwidth. The measured DSB noise-temperature and conversion-loss versus $\mathrm{LO}$ power available at $250 \mathrm{GHz}$ are shown in Fig. 8 and it is seen that the measurements level-off at an LO power of $1.5-2.0 \mathrm{~mW}$. The measured conversion loss differs by about $4 \mathrm{~dB}$ from the predicted conversion loss using the reflection algorithm [7]. Part of this $4 \mathrm{~dB}$ discrepancy is the $1.2 \mathrm{~dB}$ $\mathrm{RF}$-loss due to the uncoated sidewall $\mathrm{A}$ and the groove in wall $\mathrm{B}$. The remaining discrepancy is attributed to the non-zero impedance of the higher order harmonics, to the increase in the series resistance of the diode at high frequencies and to losses in the polyimide layer in the $\mathrm{RF}$ shorting capacitor on the CPS line. 
The measured DSB conversion loss and noise temperature between $248-265 \mathrm{GHz}$ are about $3 \mathrm{~dB}$ higher than the best room temperature waveguide mixers in this frequency range $[11,12]$. This marks the first time a planar integrated submillimeter-wave mixer with a planar diode has been designed and tested successfully at these frequencies. The receiver performance can be improved by etching via-holes type trenches in the high resistivity silicon membrane wafer around the side walls in order to eliminate the RF power loss through the uncoated wall. For SIS receivers, an RF matching network can be integrated between the detector and the dipole feeds to result in a performance with a $10-20 \%$ bandwidth. Currently, it is easy and inexpensive to array four of these receivers on a single chip. Together, the four planar receivers have a bandwidth of $10 \%$ and a sensitivity similar to that of one of the best tuned waveguide mixers.

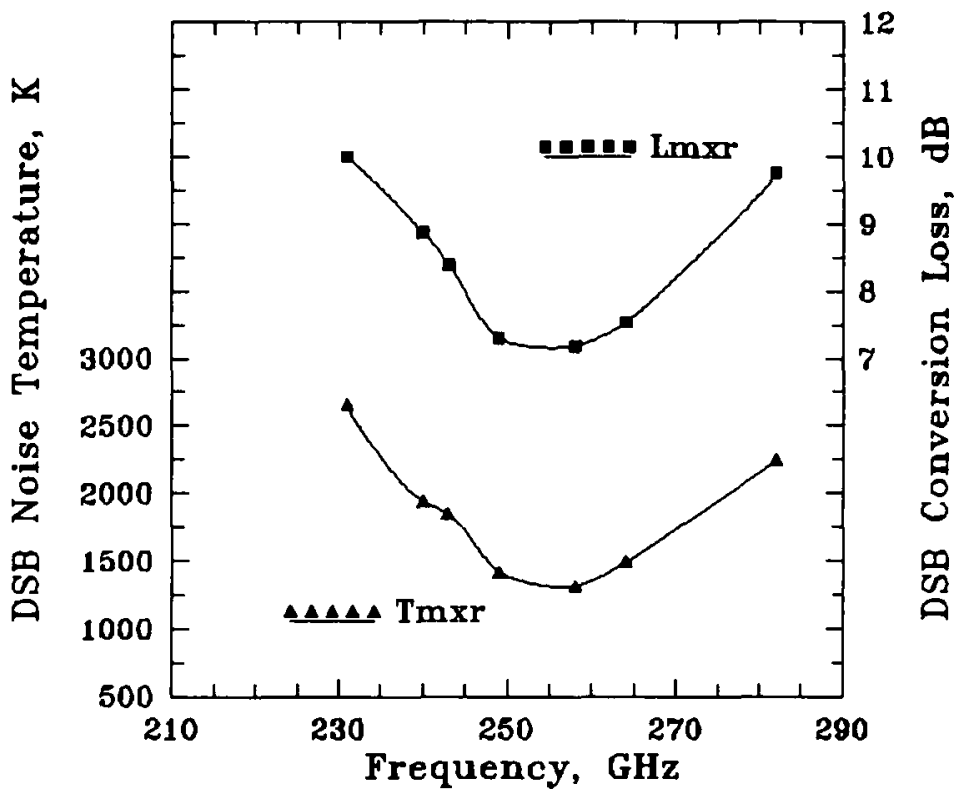

Figure 7: The measured antenna-mixer DSB noise temperature and conversion loss over the $230-280 \mathrm{GHz}$ range. 


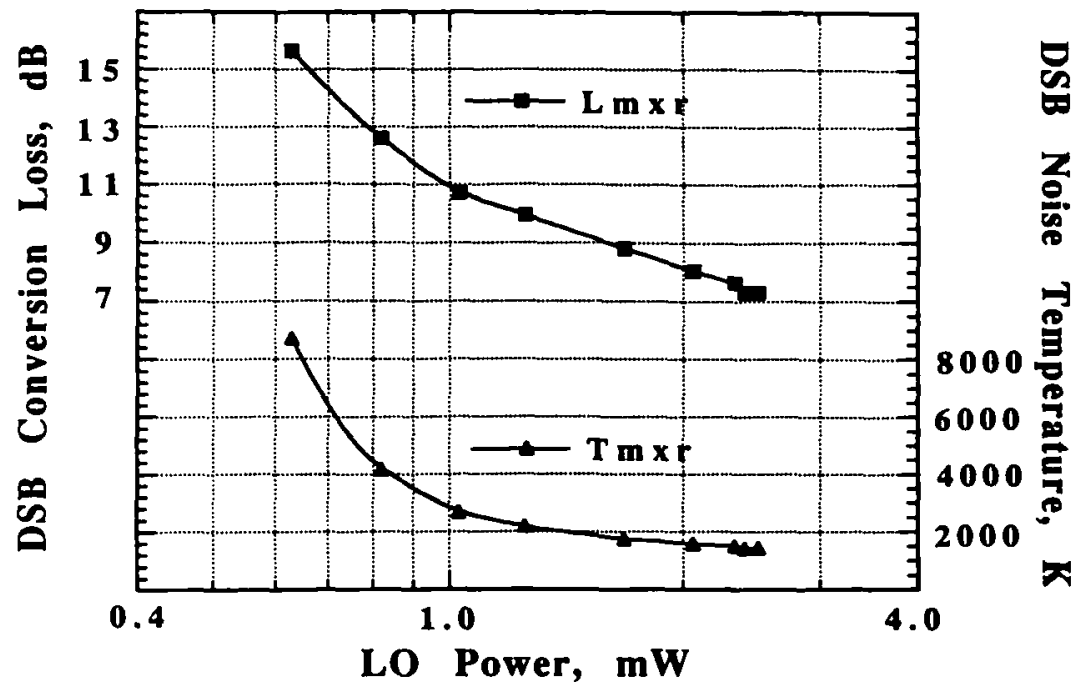

Figure 8: The measured antenna-mixer DSB noise temperature and conversion loss vs. $\mathrm{LO}$ power at $250 \mathrm{GHz}$. This is the $\mathrm{LO}$ power available at the quasi-integrated antenna aperture.

\section{ACKNOWLEDGMENTS}

This work was supported by the NASA/Center for Space Terahertz Technology at the University of Michigan, Ann Arbor, and the Solid-State Device Laboratory at the University of Virginia under the contracts NSFECS-9016063 and JPL-958202. We thank Dr. Niel Erickson, University of Massachussetts, and Jack Hardee, Jet Propulsion Laboratory-CalTech, for the use of the $280 \mathrm{GHz}$ tripler.

\section{REFERENCES}

[1] W.Y. Ali-Ahmad, W.L. Bishop, T.W. Crowe, and G.M. Rebeiz,"An 86- 106GHz Quasi-Integrated Low Noise Schottky Receiver," To appear in the April 1993 issue of the IEEE Trans. Microwave Theory Tech.

[2] G.V. Eleftheriades, and G.M. Rebeiz, "Design and Analysis of QuasiIntegrated Horn Antennas for Millimeter- and Submillimeter-wave Applications," To appear in the May 1993 issue of the IEEE Trans. Microwave Theory Tech. 
[3] G.M. Rebeiz, D.P. Kasilingan, P.A. Stimson, Y. Guo, and D.B. Rutledge, "Monolithic millimeter-wave two-dimensional horn imaging arrays," IEEE Trans. Antennas Propagat., vol AP-38, pp. 1473-1482, Sept. 1990.

[4] W.Y. Ali-Ahmad, G.V. Eleftheriades, L.P. Katehi, and G.M. Rebeiz, "Millimeter-Wave Integrated Horn Antennas, Part II: Experiment," IEEE Trans. Antennas Propagat., vol. AP-39, pp. 1582-1587, Nov. 1991.

[5] Duroid is a trademark of Rogers Corporation. We thank Rogers Co. for the donation of the substrate.

[6] T. Newman, W.L. Bishop, K.T. Ng, and S. Weinreb, "A Novel Planar Diode Mixer for Submillimeter-Wave Applications," IEEE Trans. Microwave Theory Tech., vol. MTT-39, pp. 1964-1971, Dec. 1991.

[7] D.N. Held and A.R. Kerr, "Conversion loss and noise of microwave and millimeter-wave receivers: Part I-Theory; Part II-Experiment," IEEE Trans. Microwave Theory Tech., vol. MTT-26, pp. 49-61, Feb. 1978.

[8] C.C. Ling, and G.M. Rebeiz,"A Wide-Band Monolithic Quasi-Optical Power Meter for Millimeter- and Submillimeter-Wave applications," IEEE Trans. Microwave Theory Tech., vol. MTT-39, pp. 1257-1261, Aug. 1991.

[9] M.N. Afsar, "Dielectric Measurements of Millimeter-Wave Materials," IEEE Trans. Microwave Theory Tech., vol. MTT-32, pp. 1598-1609, Dec. 1984.

[10] J.M. Dutta, C.R. Jones, and H. Davé, "Complex Dielectric Constants for Selected Near-Millimeter-Wave Materials at 245GHz," IEEE Trans. Microwave Theory Tech., vol. MTT-34, pp. 932-936, Sep. 1986.

[11] J.W. Archer, "All Solid-State Low Noise Receivers for 210-240 GHz," IEEE Trans. Microwave Theory Tech., vol. MTT-30, pp. 1247-1252, Aug. 1982.

[12] N.R. Erickson, "A Very Low-Noise Single-Sideband Receiver for 200260GHz," IEEE Trans. Microwave Theory Tech., vol MTT-33, pp. 11791188, Nov. 1985. 